\title{
Evacuation Simulation of Counter-current Behavior Using Agent Model for Pedestrian Dynamics
}

\author{
Xiaoan Zhao ${ }^{\mathrm{a}}$,Yanyan You ${ }^{\mathrm{a}, \mathrm{b}}$, Ying Zhang ${ }^{\mathrm{b}, \mathrm{c}}$ \\ ${ }^{a}$ School of Computer Science and Engineering, Hebei University of Technology, Tianjin, China \\ ${ }^{b}$ School of Electrical Engineering and Automation, Hebei University of Technology, Tianjin, China
}

\begin{abstract}
It is an important issue that all occupants should be able to evacuate to safety from sports stadium under emergency. The behavior of occupants is quite complex, and individual behavior will greatly affect the result of the evacuation. Such as the phenomenon of counter-current behavior, sub-group behavior, following behavior and so on. In this paper, we mainly study the counter-current behavior, and a system simulation model is presented, which based on the agent technology. And in order to study the effect on evacuation, two cases have been studied to analyze the impact of evacuation. Simulation processes and results display that the counter-current behavior will be harmful for the movements of people. Simulation processes and results display that the model is very close to real movements of people.
\end{abstract}

Index Terms:Pedestrian evacuation; microscopic Model; Agent; counter-current

(C) 2011 Published by MECS Publisher. Selection and/or peer review under responsibility of the International Conference on EBusiness System and Education Technology

\section{Introduction}

In recent years, the socio-economic and sports architecture have been experiencing surprisingly rapid growth, and there are more people in the building. In order to simulate the behavior of evacuation, we not only consider the environment, but also consider the social relationship. And the work on human behavior has attracted a lot of researchers, such as Mr. J. D. Sime who has made a lot of useful studies on the affiliation model and movement with a panic [1].

It is not easy to predict evacuation performances for sports stadium with complex layouts. The situation will become even more sophisticated when considering the interaction between occupants and other factors. The development of simulation models makes it possible to find out relevant quantity distribution in field without practical experiments. The investigation on human psychology and behavior play an important role in the research on evacuation efficiency. In this paper, a basic Agent-based evacuation models is applied to study the typical behavior of counter-current and to investigate the disadvantages of the evacuation caused by the kin behavior.

\section{Model}

Under the guidance of a complex adaptive system theory, the Agent-based evacuation models combined cellular automata model and computer simulation techniques to study complex systems. It is an effective method.

\section{A. Individual Decision-Making Model}

Agent is an emerging technology in the field of artificial intelligence and computer software. It has the following features [2]:

- Autonomous: Agent can conduct some control of their acts and internal state, it can operate without direct intervention by human or other agents.

* Corresponding author.

E-mail address: ${ }^{\mathrm{a}} \mathrm{zhxa} 2006 @$ yahoo.com.cn, ${ }^{\mathrm{b}}$ wsyouyan@163.com, ${ }^{\mathrm{c}}$ sbczhang@hehut.edu.cn 
- Social: Agent can communicate with other agents.

- $\quad$ Reactive: They can sense the environment and respond promptly to environmental changes.

The agent can not only reflect the environment, but also take the initiative to achieve the goal. And it can be driven by various factors with time flow. The individual decision-making model (as shown in Fig.1) is proposed.



Figure1. Individual decision-making model

As shown in the agent model, we know that within a certain viewing range the agent could take actively by individual perception, judgment and response. And in this paper we introduce the conception of neighborhood to simulate the effect of local visual field. Around each agent, there exists a neighborhood, which could simulate the occupant's view field in some case. The current state parameters of the gird within the neighborhood will affect the agent's decision in the next step. And in the model, we select Moore neighborhood [3] (as shown in Fig.2) as the neighborhood of each individual. The current behavior of each individual is totally decided by the environmental parameters within the neighborhood and the cooperation with other agents. It is reasonable that the movement of people in the building is normally based on the local environment rather than the whole building.

Figure2. Moore

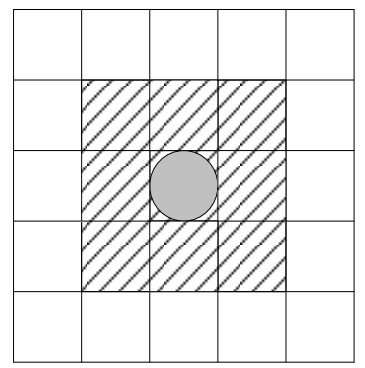

\section{B. Gird model}

In this paper we will use the CA model which is a two-dimensional grid, CA-based pedestrian flow model has been defined on a discrete $M \times M$ cell grid in a two-dimensional system, and the size of a grid corresponds to $0.4 m \times 0.4 m$, which is the typical space occupied by an occupant in a dense crowd [4]. Each sell can either be occupied by one pedestrian or empty. The simulation procedure is divided into discrete time steps. In every step, the individual can only select a cell. It means that the evacuation model generates all the occupants in the environment and produces the simulation for each occupant at each time step, the agent may choice made concerning pause or movement. And the agent need to select the possible moving directions in the 9 directions [5] (as shown in Fig.3).

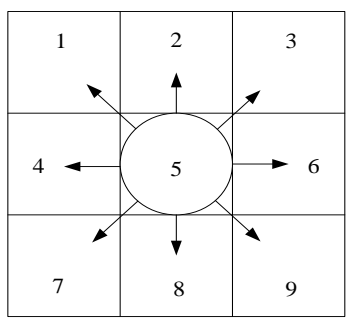

Figure3. Possible moving directions of each agent 


\section{Individual behavior model}

The behavior response to the emergence is complex with changing situations. In order to understand the typical behavior, we should consider the influence on environment and the socio-psychological between the individuals.

During evacuation the kin-behavior is very important, such as : the behavior of going with the crowd (means one gives up his own mind and takes the actions as the most people behavior around), get together (occupants are willing to close with the crowd before evacuation), counter-current and entering the room again (the main purpose maybe saving personal belonging, help other people and so on), the counter-current is normal, such as, parents often backtrack if their children are lost or people go back to retrieve their valuables, but the backtrack behavior will bring negative influences and be harmful to other occupant, so we mainly research the counter-current behavior in two aspects in this paper: First, the children are stay in the room until their parents are coming, and it means that the people is stay in place until others to help them; Second, the children will move to the exit without waiting for their parent.

\section{Math model}

The agent decision model was constructed through the integration of intelligent simulation methods including agent technology and rule reasoning etc. As a representation of the occupants, the agent makes decision on behavior at each time instant according to certain rules [6].

To different occupants the behavior will be different, we can study the behavior for different agents [7]. And the math model will be different, such as the sub-groups which will get together before simulation, but the counter-current occupants will backtrack to the Sports Stadium, in this paper, we will study the counter-current behavior.

At each time step, each agent need to decide the target exit, the optimal or sub-optimal direction, but to the counter-current agent before moving out the stadium. They need to decide the backtrack behavior, in order to help other people or get back their values which are lost in the stadium.

\section{1) The backtrack action}

In this paper we mainly research the counter-current behavior. For the follower occupant, the behavior can be divided into two categories.

One is that the follower will move to the exit without consider the leader, if the distance between the leader and the follower is less than D. The other is that the follower will take back behavior until the distance reaches the give value D. But the backtracking behaviors are all affected by the attraction $G_{g}$ of the counter-current individual.

$G_{g}$ could be expressed with the following formula:

$$
\begin{aligned}
& G_{g}^{\prime}=\sqrt{\left(x_{L}{ }^{i}-x_{F}^{j}\right)^{2}+\left(y_{L}{ }^{i}-y_{F}^{j}\right)^{2}}
\end{aligned}
$$

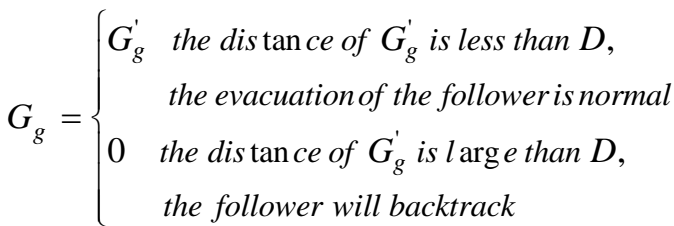

$D=\lambda \times n,\left(x_{L}^{j}, y_{L}^{j}\right)$ is the location of the leader, $\left(x_{F}^{i}, y_{F}^{i}\right)$ is the location of the follower, $\lambda$ is the size of the grid, $n$ is the coefficient of the regulation distance.

\section{2)The wait behavior}

The leader will take different actions, as define in the following function.

$$
T_{g}=\left\{\begin{array}{c}
0 \quad \text { the dis } \tan \text { ce of } g_{g}^{\prime} \text { is less than } d, \\
\text { the leader will moving } \\
2 \text { the dis } \tan \text { ce of } g_{g}^{\prime} \text { is } l \arg \text { e than } d, \\
\text { the leader will waiting }
\end{array}\right.
$$

The function of $g_{g}^{\prime}$ is the same to $G_{g}^{\prime}$.

\section{3) The general action}


The occupant in order to the safe area, they need select the target exit and the move direction [8], and the process is the same to every occupant.

According to the process of evacuation, the target exit is abstracted as $E_{t+1}$, and it could be expressed with the following formula:

$$
E_{t+1}=f\left(P_{r t}+O_{r t}+D_{r t}+d_{t}^{\prime}+p_{t}^{\prime}+o_{t}^{\prime}\right)
$$

Where $P_{r t}$ is the number of people in the viewing range. $O_{r t}$ is the number of obstacle in the viewing range. $D_{r t}$ is the function of distance and $d_{t}^{\prime}$ is the distance between leader and follower. $p_{t}^{\prime}$ is the occupant density of viewing. $o_{t}^{\prime}$ is the obstacle density of viewing.

The function of $P_{r t}$ is shown as follow:

$$
P_{r t}=f\left(p_{t}\right)
$$

Where $p_{t}$ is the number of the occupants in the viewing .

$$
P_{t}=\sum_{\left(x^{\prime}, y^{\prime}\right) \in R} f_{1}\left(x^{\prime}, y^{\prime}\right)
$$

While $f_{1}\left(x^{\prime}, y^{\prime}\right)= \begin{cases}1 & \text { occupied byoccupant } \\ 0 & \text { empty }\end{cases}$

The function of $O_{r t}$ is shown as follow:

$O_{r t}=f\left(o_{t}\right)$

Where $o_{t}$ is the number of the obstacle in the viewing range.

$o_{t}=\sum_{\left(x^{\prime}, y^{\prime}\right) \in R} f_{2}\left(x^{\prime}, y^{\prime}\right)$

While $f_{2}\left(x^{\prime}, y^{\prime}\right)= \begin{cases}1 & \text { occupied byobstacle } \\ 0 & \text { empty }\end{cases}$

The density of people and obstacle are shown as follow:

$p_{t}^{\prime}=\frac{p_{t}}{N U M}$

$o_{t}^{\prime}=\frac{o_{t}}{N U M}$

$N U M$ is the number of the grid in the viewing.

The function of $D_{r t}$ is shown as follow:

$D_{r t}=f\left(d_{t}\right)$

Where $d_{t}$ is the function of the distance, and $d_{t}$ could be expressed with the following formula:

$$
d_{t}=\sqrt{\left(x-x_{r t}\right)^{2}+\left(y-y_{r t}\right)^{2}}
$$

Where $(x, y)$ is the location of the 9 moving directions, $\left(x_{t}, y_{t}\right)$ is the location of the exit.

$d_{t}^{\prime}$ is the distance between the leader and the follower:

$d_{t}^{\prime}=\sqrt{\left(x_{1}^{\prime}-x_{t}^{\prime}\right)^{2}+\left(y_{1}^{\prime}-y_{t}^{\prime}\right)^{2}}$ 
Where $\left(x_{1}^{\prime}, y_{1}^{\prime}\right)$ is the location of the leader, $\left(x_{t}^{\prime}, y_{t}^{\prime}\right)$ is the location of the follower.

There is a probability between the optimal and sub-optimal direction. Such as we set the probability of the optimal direction as $90 \%$, then the probability of the sub-optimal is $10 \%$. The agent will select the direction between the optimal and the sub-optimal according to the probability.

\section{Simulation and result analysis}

\section{E. Simulation}

This article considers that occupants are familiar with the architectural environment. Occupants are generated randomly before evacuation starts. The initial location and inherent qualities like gender are randomly given to each individual. Then some evacuation properties relevant to qualities are assigned according. And different individual will have different properties.

In this paper, we will use the framework as shown in Fig. 4.

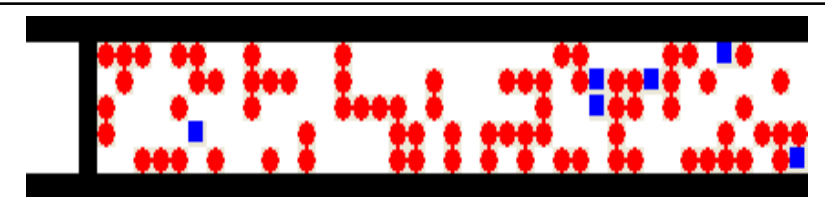

Figure4 The framework of simulation evacution model for counter-current behavior

As shown in Fig.4, the character of the environment and the occupants are as follow:

- The studied building covers an area of $17.2 \mathrm{~m} \times 2.8 \mathrm{~m}$.

- There are 82 pedestrians in the building, and in order to reflect the phenomenon of counter-current we set the individual with blue color in the building (as shown in Fig.5 (a)), and the shape of counter-current is rectangle.

- The stadium is one-way corridor, and the direction of movement is right, and the width of the corridor is 5 gird.

- In order to simulate the counter-current behavior, in this paper, we use two cases to simulate the behavior.

1) Case 1: The backtrack behavior

The total number of the agent is 82 and the counter-currents have 6 occupants. We define the backtrack agent as the follower, and the other one is the leader.



(b)

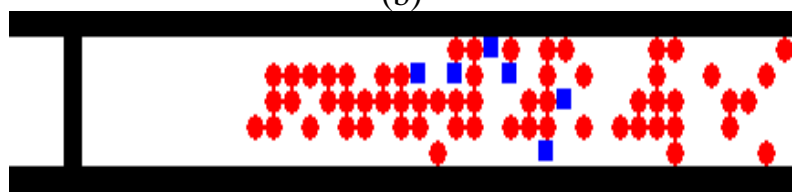

(c)

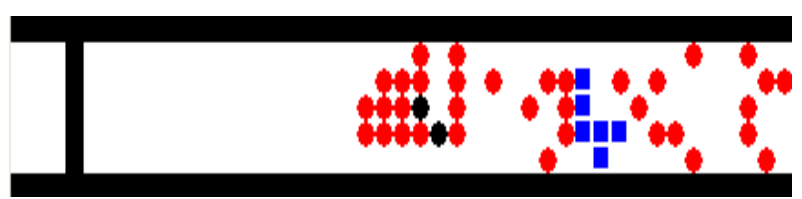

(d) 


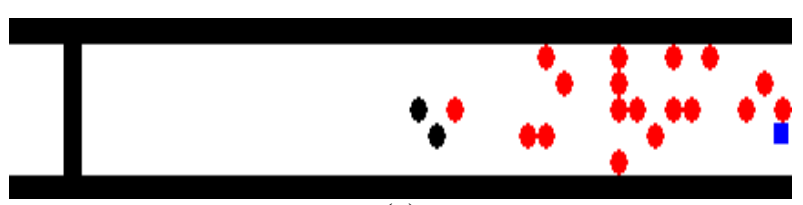

(e)

Figure5 Evacuation of counter-current behavior with moving leader

From Case 1 we can see that:

a) During the evacuation the non-counter move to the exit spontaneously (as shown in Fig.5(b), (c) and (d)).

b) In the evacuation process, the leader moves to the exit and the followers have move to the opposite direction which is away from the exit, there is an apparent counter-current behavior (as shown in Fig.5(b) and (c)).

c) An clogging behavior can be observed (as shown in Fig.5(b)and (c)), and with the developing of clogging behavior the crowd becomes denser and denser.

d) In the evacuation process, with the counter-current, An blocking behavior can be observed (as shown in Fig.5(b)and (c)).

e) The leaders move to the exit without waiting the follower occupant (as shown in Fig.5 (b), (c) and (d)).

With different capacities of individuals, there are casualties as the time flow (as shown in Fig.5 (d) and (e)).

Through changing the number of the counter-current occupants, we can see that when the number is different, the evacuation time and steps are different, and the conclusion is given in table I.

TABLE I. EVACUATION TIME AND STEPS

\begin{tabular}{ccc}
\hline $\begin{array}{c}\text { Counter-current } \\
\text { number }\end{array}$ & $\begin{array}{l}\text { Evacuation } \\
\text { time(s) }\end{array}$ & $\begin{array}{l}\text { Evacuation } \\
\text { steps }\end{array}$ \\
3 & 48.9 & 2034 \\
6 & 59.6 & 2214 \\
12 & 76.6 & 3198 \\
\hline
\end{tabular}

From the table we can see that as the increase of the counter-current number, non-linear increase can be shown in the evacuation time and steps, and the efficiency of evacuation is decrease.

2) Case 2: The waiting behavior

The occupant number is 82 and counter-currents is 6 occupants (as shown in Fig.6), which is the same to the backtrack behavior. But in the simulation we mainly study the waiting behavior of the leader, who will wait the follower until the distance achieves the given value (as shown in Fig.6).

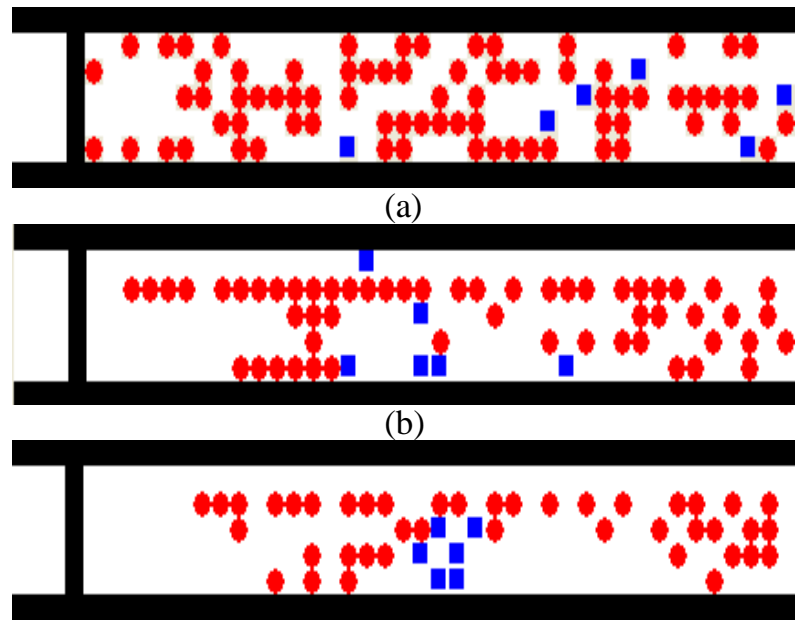

(c) 


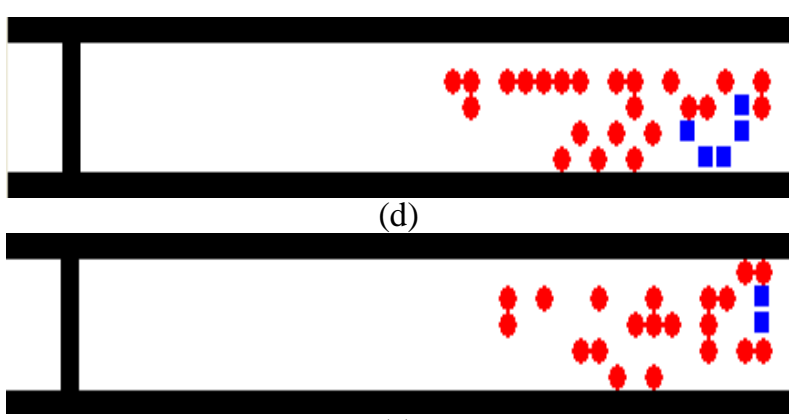

(e)

Figure6 Evacuation of counter-current behavior with waiting leader

Form the case, we can see that the result is the same to case 1, except that during the simulation, the leader will wait in the location until the distance between the leader and the follower achieves the given value, and then the leader will begin to move to the exit, and the evacuation process is normal.

When the number of the counter-currents is different, the result must be different, as shown in table 2 .

TABLE II. EVACUATION TIME AND STEPS

\begin{tabular}{ccc}
\hline $\begin{array}{c}\text { Counter-current } \\
\text { number }\end{array}$ & $\begin{array}{c}\text { Evacuation } \\
\text { time(s) }\end{array}$ & $\begin{array}{c}\text { Evacuation } \\
\text { steps }\end{array}$ \\
3 & 52.1 & 2134 \\
6 & 67.9 & 2336 \\
12 & 127.5 & 3971 \\
\hline
\end{tabular}

From the table we know that as the increase of the counter-current number, the phenomenon of non-linear increases can be shown in the evacuation time and steps.

\section{F. Result analysis}

From the two cases, we get the result when there is the behavior of counter-current, the efficiency of evacuation is decrease. As shown in table 3 , the counter-current number is 6 or 0 .

TABLE III. EVACUATION TIME AND STEPS

\begin{tabular}{cccc}
\hline $\begin{array}{c}\text { Counter-current } \\
\text { number }\end{array}$ & $\begin{array}{c}\text { The leader } \\
\text { state }\end{array}$ & $\begin{array}{c}\text { Evacuation } \\
\text { time }\end{array}$ & $\begin{array}{c}\text { Evacuation } \\
\text { steps }\end{array}$ \\
0 & ------- & 52.3 & 1906 \\
6 & move & 59.6 & 2214 \\
6 & wait & 67.9 & 2336 \\
\hline
\end{tabular}

From the table, we can see that when there are counter-currents, the evacuation time and steps are non-linear increase and when the leader is waiting in the place, the evacuation time and steps are corresponding to increase in time. Thus, the above data showed that the presence of counter-current not only hamper the normal evacuation, but also extend their evacuation time, and seriously disrupting the orderly, making the evacuation time and steps increase. Therefore, the counter-current phenomenon reduced the efficiency of the evacuation.

Through the simulation we also get a result that with different capacities of individuals, the casualties can be observed as time goes on. And the more number of the counter-current, the more casualties can be shown in the building. From the result, we concluded that we should try to avoid the counter-current phenomenon in the actual evacuation. 


\section{Conclusions}

With the increase of processor power and memory capacity of modern computers, it becomes more and more popular and effective to conduct simulation experiments on computer. In this study, an evacuation simulation model based on individual decision-making is proposed, which aims at simulating counter-current phenomenon. By the simple rules, the model consider the social force among pedestrians (such as the attraction between the pedestrian, and mainly to the counter-current pedestrian).Through the simulation we concluded that the counter-current phenomenon may reduce the efficiency of evacuation. And with the increase of the counter-current occupants, there will non-linear increase in evacuation time.

From the simulation, we can see that the model is easy to operate and verify, and the results are demonstrated to be qualitatively correct, and the model is useful for the reality. In future work, we would like to perfect the model and the evaluation methods of the evacuation efficiency for architectural plans.

\section{References}

[1] L.Z.Yang, D.L.Zhao, J.Li,T.Y.Fang.Simulation of the kin behavior in building occupant evacuation based on Cellular Automaton, Building and Environment 40(2005),pp. 411-415 (in Chinese).

[2] Jiang Yunliang and Xu Congfu, "Intelligent Agent and Multi-Agent System”, Application Research of Computers, 2003.4 (in Chinese).

[3] J. Neumann In: A.W. Burks, Editor, Theory of Self-Reproducing Automata, University of Illinois Press, Urbana (1966).

[4] C. Burstedde, K. Klauck and A. Schadschneider et al, Simulation of pedestrian dynamics using a two dimensional cellular automaton, Physica, A 295 (324) (2001), pp. 507-525.

[5] Hao Yue,Hongzhi Guan,Juan Zhang,Chunfu Shao.Study on bi-direction pedestrian folw using cellular automata simulation,Physica A 389(2010), pp.527-539 (in Chinese).

[6] H.E. Nelson and H.A. MacLennan, Emergency movement, The SFPE Handbook of Fire Protection Engineering (2nd ed) (1996), pp. 3-286-3-295.

[7] Zheng Xiaoping,Li Wei,Guan Chao.Simulation of evacuation processes in a squre with a partition wall using a cellular automaton model for pedestrian dynamics, Physica A 389(2010)2177-2188 (in Chinese).

[8] Ying Zhang, Xiaoan Zhao, Lei Zhang, Xifa Huang. Study on the Agent-based Evacuation Simulation Models for Large Public Buildings[J]. 2009 Second International Conference on Intelligent Networks and Intelligent Systems, $2009: 522 \sim 534$. 